\section{Voxel based analyses of diffusion tensor imaging in Fabry disease}

\author{
J Albrecht, P R Dellani, M J Müller, I Schermuly, M Beck, P Stoeter, \\ A Gerhard, A Fellgiebel
}

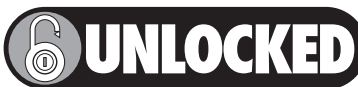

This paper is freely available online under the BMJ Journals unlocked scheme, see http://jnnp.com/info/unlocked.dtl
See end of article for authors' affiliations

Correspondence to:

Dr A Fellgiebel, Department of Psychiatry, University Hospital of Mainz, Untere Zahlbacher Str 8, 55131 Mainz, Germany; fellgiebel@psychiatrie.klinik. uni-mainz.de

Received 10 December 2006 Revised 5 March 2007 Accepted 14 March 2007

Published Online First 20 April 2007
Background: Fabry disease (FD) is a lysosomal storage disorder associated with marked cerebrovascular disease. Conventional MRI shows an extensive load of white matter lesions (WMLs) which may already be present at an early stage in the disease.

Objective: Investigator independent and sensitive quantification of structural changes in the brain in clinically affected men and women with FD.

Methods: We performed a voxel based analysis of diffusion tensor images (DTI) in 25 patients with FD and 20 age matched normal controls.

Results: DTI revealed significant increases in cerebral white matter mean diffusivity (MD) in patients with FD, which were pronounced in the periventricular white matter. Even the subgroup of patients without significant WMLs load $(n=18)$ showed increased diffusivity in the cerebral white matter. In gray matter areas, MD elevation was detected only in the posterior part of the thalamus, independent of the visible pulvinar alterations on $\mathrm{Tl}$ weighted images. Voxel based fractional anisotropy measurements did not differ significantly between patients and controls.

Conclusions: The present study demonstrates the clinical feasibility of voxel based analysis of DTI as a sensitive tool to quantify brain tissue alterations in FD. The pattern of increased brain tissue diffusivity is probably due to microangiopathic alterations, mainly affecting the long perforating arteries.
$\mathrm{F}$ abry disease (FD) is a rare X-linked hereditary lysosomal storage disease. ${ }^{1}$ Deficiency of $\alpha$-galactosidase A activity leads to accumulation of neutral glycosphingolipids, particularly in vascular endothelial and smooth muscle cells of various organs. ${ }^{2}$ Together with progressive renal and cardiac dysfunction, clinical CNS involvement mainly due to cerebral vasculopathy leading to serious complications such as stroke at an early age has been confirmed in clinical studies ${ }^{3}$ and by neuroimaging. ${ }^{5-10}$ The most prominent brain structural findings in FD are severe progressive white matter lesions (WMLs) that are detectable by $\mathrm{T} 2$ and FLAIR weighted images using conventional MRI. ${ }^{5}{ }^{7} 10-12$

Focusing on WMLs and quantifying structural cerebrovascular involvement by visual rating scales on conventional MRI is compromised by limited accuracy. ${ }^{13}{ }^{14}$

Investigation of the structure of the brain, in particular the detection of typical patterns of brain structural involvement as well as assessment of the natural course of these affections, is important for a better understanding of the pathophysiology of the brain and to study the potential effects of new treatment options, such as enzyme replacement therapy (ERT) in FD.

There is evidence that diffusion tensor imaging (DTI) is more accurate and more sensitive in quantifying structural brain alterations than other assessments in conventional magnetic resonance techniques. ${ }^{6}{ }^{15-17}$ The principal parameters derived from diffusion tensor analysis are fractional anisotropy (FA) and mean diffusivity (MD). Reduced water diffusion parallel to axonal tracts represented by FA is indicative of axonal degeneration. ${ }^{18}$ Furthermore, MD, which measures randomised mean water diffusion, is a representative DTI parameter for ultrastructural brain tissue alterations. ${ }^{19}$ Thus DTI provides a promising tool for detection of early ultrastructural cerebral changes in FD.

The use of diffusivity measurements in patients with FD has been demonstrated previously. One study showed global elevation of the average diffusion constant in cerebral tissue from men with FD. ${ }^{20}$ Moreover, analysing DTI with regions of interest (ROI), we previously showed significant elevations in white matter diffusivity (MD), even in those FD patients without significant WMLs. ${ }^{6}$ However, ROI based analysis is time consuming, investigator dependent and usually does not cover the whole brain.

To investigate the feasibility of investigator independent structural data processing procedures which cover the whole brain, we analysed DTI data from patients with FD using a voxel based approach. In this way, structural differences in the brain can be detected by voxel wise comparison of normalised DTI maps between patient and control groups.

\section{SUBJECTS AND METHODS}

\section{Patients}

We enrolled 25 clinically affected FD patients ( 10 men, mean age 36.1 (12) years; 15 women, mean age 36.7 (11.5) years) and 20 age matched healthy controls ( 12 men, mean age 34.4 (10.2) years; 8 women, mean age 36 (9.4) years). Twenty-two patients and 18 controls were part of our previously published ROI based study. ${ }^{6}$ All patients were recruited at the Children's Hospital, University of Mainz. For the enzymatic and molecular diagnosis of FD, standard methods were used, as generally recommended. ${ }^{21}$ The average clinical stage of patients with FD was moderate. None of the patients had end stage renal failure or had undergone renal dialysis. Table 1 shows the patient characteristics. Inclusion criteria have been described previously. ${ }^{611}$ At the time of assessment, 20 patients were receiving ERT or were about to start ERT (five patients, two patients had

Abbreviations: DTI, diffusion tensor imaging; ERT, enzyme replacement therapy; FA, fractional anisotropy; FD, Fabry disease; FLAIR, fluid attenuated inversion recovery; $M D$, mean diffusivity; ROI, regions of interest; WML, white matter lesion 
received previous ERT). Age matched controls were recruited by advertisement and underwent a structured interview and physical examination to exclude cerebral diseases as well as clinically manifest psychiatric disorders or significant organic dysfunction. The study was approved by the local ethics committee and all subjects gave written informed consent.

\section{Magnetic resonance data acquisition}

All data were obtained on a 1.5 Tesla system with gradients of $40 \mathrm{mT} / \mathrm{m}$ (Magnetom Sonata; Siemens, Erlangen, Germany). Apart from the acquisition of routine Tl (TR/TE: $600 \mathrm{~ms} / 25 \mathrm{ms,}$ matrix $256 \times 256$ ), PD/T2 weighted (TR/TEl/TE2: $4500 \mathrm{~ms} /$ $15 \mathrm{~ms}, 100 \mathrm{~ms}$, matrix $256 \times 256)$ and fluid attenuated inversion recovery (FLAIR) weighted (TR/TE: $9000 \mathrm{~ms} / 108 \mathrm{ms,} \mathrm{slice}$ thickness $6 \mathrm{~mm}$, matrix $512 \times 448)$ images, and three dimensional magnetisation prepared rapid gradient echo (MP-RAGE) (TR/TE: $1900 \mathrm{~ms} / 16 \mathrm{~ms}$, matrix $512 \times 512$ ) data sets, we used a transverse diffusion weighted, single shot, spin echo, echo planar based sequence with gradients along six non-collinear directions (TR/TE 8000/105 ms, b $=0$ and $1000 \mathrm{~s} / \mathrm{mm}^{2}$, matrix $128 \times 128$, slice thickness $3 \mathrm{~mm}$ without gap (voxel size $1.8 \times 1.8 \times 3.0 \mathrm{~mm}))$ and six averages. The transverse slices were aligned to the AC-PC line (anterior-posterior commissure) and covered the whole brain, except the top $6 \mathrm{~mm}$.

\section{White matter lesion classification}

WML load was classified on FLAIR images using a simplified, previously described, visual rating scale $^{11}: 0=$ no WML, $1=$ mild WMLs (up to 5 single, non-confluent lesions), $2=$ significant WMLs (more than 5 or confluent).

\section{DTI data post-processing}

Magnetic resonance DTI datasets were transferred to a Linux $\times 86$ Workstation for post-processing. The diffusion tensors were computed using an inhouse programme based on the algorithm of Basser et al. ${ }^{22}$ Decomposition of the diffusion tensor (D) in an eigen system was accomplished by symmetric bidiagonalisation followed by QR reduction, implemented using the respective functions, as supplied with the GSL (Free Software Foundation, GSL-GNU Scientific Library, 2001; http:www.gnu.org/software/gs/ accessed 28 May 2005). Mean diffusivity $(\mathrm{MD})$ is the mean of the diffusion tensor eigen values $\left[\left(D_{x x}+D_{y y}+D_{z z}\right) / 3\right.$ given in $\left.\mathrm{mm}^{2} / \mathrm{s}\right]$. Fractional anisotropy $(F A)$ is the $\mathrm{SD}$ of eigen values from the MD normalised by square norm of the eigen values. ${ }^{22}$ Both indices were plotted voxel by voxel as MD and FA index maps.

Table 1 Clinical characteristics of the patients and controls

\begin{tabular}{|c|c|c|}
\hline & Fabry disease & Controls \\
\hline $\mathrm{n}$ (women) & $25(15)$ & $20(8)$ \\
\hline Age $(y)^{*}$ & $\begin{array}{l}36.4(11.5) \\
(19-55)\end{array}$ & $\begin{array}{l}35.1(9.7) \\
(22-55)\end{array}$ \\
\hline Cerebrovascular events & $n=5(20 \%)$ & 0 \\
\hline Neuropathic pain & $n=20(80 \%)$ & 0 \\
\hline Angioceratoma & $n=13(52 \%)$ & 0 \\
\hline Cardiac dysfunction & $\mathrm{n}=20(80 \%)$ & 0 \\
\hline Cardiomyopathy & $n=13(52 \%)$ & \\
\hline Arrhythmias & $\mathrm{n}=7(28 \%)$ & \\
\hline Left ventricular hypertrophy & $\mathrm{n}=1(5 \%)$ & \\
\hline Renal dysfunction & $n=20(80 \%)$ & 0 \\
\hline Renal insufficiency & $\mathrm{n}=4(16 \%)$ & \\
\hline Proteinuria & $\mathrm{n}=16(64 \%)$ & \\
\hline Serum creatinine $(\mathrm{mg} / \mathrm{dll})^{*}$ & $0.92(0.41)$ & \\
\hline
\end{tabular}

Image processing: voxel based DTI

A voxel based analysis of parameters derived from magnetic resonance DTI (MR-DTI) was used and based on the voxel based morphometry implemented with SPM2 software optimised for white matter. ${ }^{23}$ Firstly, a template was created by normalising all images to standard space (EPI template, International Consortium for Brain Mapping) which approximates the Talairach and Tournoux space. ${ }^{24}$ In a second step, all images were normalised using the customised template. For the creation of the customised template, all non-diffusion weighted images from the DTI datasets (B0 images) were normalised to the standard anatomical space. Every B0 image was segmented in its gray and white matter volumes. All white matter volumes were normalised to the white matter template accompanying SPM2 and the resulting normalisation parameters were applied to the respective raw $\mathrm{B} 0$ images. A new $\mathrm{B} 0$ template was created by averaging all normalised B0 images. All normalised B0 images were again segmented and the resulting volume images were averaged.

The customised gray matter, white matter and CSF templates were set up as the a priori brain tissue distribution probability maps used in the segmentation procedure. All B0 images were segmented using the customised Bayesian Priors. The white matter image volumes were normalised to the customised white matter template, and the respective volume images of FA, $\mathrm{MD}$ and B0, lying in native space, were transformed accordingly to the resulting normalisation parameters.

\section{Image segmentation and threshold}

Partial volume effects can be reduced by segmenting the normalised FA and MD images into CSF, gray matter and white matter compartments. ${ }^{25}$ Segmentation algorithms for defining gray and white matter were based on a probability of greater than 0.80, according to Medina et al. ${ }^{26}$ Thus only voxels surviving this threshold were included in the group analyses. The individual gray and white matter masks were then combined and applied to individual MD and FA maps.

As MD is markedly higher in CSF than in brain tissue, a threshold procedure can be used effectively to additionally eliminate partial volume effects due to CSF in the segmented MD maps. ${ }^{27-29}$ Therefore an empirically set cut off threshold of $\mathrm{MD}=1050 \times 10^{-6} \mathrm{~mm}^{2} / \mathrm{s}$, equalling approximately 3 SDs above the mean measured tissue MD value $\left(774(96) \times 10^{-6} \mathrm{~mm}^{2} / \mathrm{s}\right)$, was applied. With this conservative threshold, those critical MD values, from periventricular border zones in particular, were excluded, which in part were significantly elevated in patient versus control comparisons.

As FA is even more susceptible to errors arising from partial volumes, ${ }^{30}$ a cut off was applied for combined gray and white matter segmented FA maps, excluding all FA values under 0.25. FA of CSF and cortical gray matter is typically below $0.2 .^{31}$ The processed FA and MD maps were then smoothed using an isotropic Gaussian filter (full width half maximum $10 \mathrm{~mm}$ ).

\section{Calculation of mean global FA and MD}

Independent of voxel based DTI, mean global FA and MD values were directly calculated from original diffusion tensor images after using the described FA and MD thresholds and gray and white matter segmentation.

\section{Statistical analysis}

Voxel wise group differences in MD and FA were calculated using unpaired Student's t tests in SPM2. An uncorrected $p<0.001$ on the voxel level was selected as a threshold of significance. The amount of adjacent voxels with significant MD elevations was represented in total cluster size. Determination of the location of clusters demonstrating 
Table 2 White matter lesion quantification in patients with Fabry disease and in controls

\begin{tabular}{|c|c|c|c|c|c|c|c|c|}
\hline \multirow{2}{*}{$\begin{array}{l}\text { WML } \\
\text { classification }\end{array}$} & \multicolumn{2}{|c|}{$\begin{array}{l}\text { Male Fabry } \\
\text { patients }\end{array}$} & \multicolumn{2}{|c|}{$\begin{array}{l}\text { Female Fabry } \\
\text { patients }\end{array}$} & \multicolumn{2}{|c|}{ Male controls } & \multicolumn{2}{|c|}{ Female controls } \\
\hline & $\mathbf{n}$ & Age & $\mathbf{n}$ & Age & $n$ & Age & $n$ & Age \\
\hline No WML & 4 & $26.8(7.0)$ & 7 & 30.7 (11.0) & 9 & $34.1(8.3)$ & 7 & $34.1(8.4)$ \\
\hline Mild WMLs & 2 & $30.0(4.0)$ & 5 & $39.4(9.6)$ & 3 & 35.3 (17.1) & 1 & 49.0 \\
\hline Significant WMLs & 4 & $48.5(5.7)$ & 3 & $46.0(10.1)$ & 0 & & 0 & \\
\hline
\end{tabular}

WML, White matter lesion.

Mild WMLs, single, non-confluent bright lesions; significant WMLs, multiple (>5) or confluent lesions.

significantly different DTI values was accomplished by converting the $\mathrm{x}, \mathrm{y}$ and $\mathrm{z}$ coordinates for the peak voxel within a cluster from the Montreal Neurological Institute (MNI) coordinates used in SPM2 analyses to Talairach coordinates using MSU-MNI Space Utility software (http://www.ihb.spb.ru/ $\sim$ pet_lab/MSU/MSUMain.html). This software program also identified nearest lobar and Brodmann area locations.

Further statistical analysis was performed with SPSS software package version 12.0 (SPSS, Chicago, Illinois, USA, 2003). Descriptive data are reported as means and SD. The analysed mean global FA and MD values did not show any significant deviation from normal distribution (Komolgorov-Smirnov tests, $\quad \mathrm{>}>0.74)$; hence, parametric tests were used. Comparisons (FD vs controls) for mean global MD and FA values of gray and white matter segments of all 45 studied subjects were carried out using unpaired Student's t tests. Association of clinical features with imaging parameters of the patient group was analysed using univariate analysis (ANOVA) or binary logistic regression with age as the covariate. Spearman's rank $\left(\mathrm{r}_{\mathrm{s}}\right)$ and partial $\left(\mathrm{r}_{\mathrm{p}}\right)$ correlation coefficients were calculated for correlations of WMLs load, age and global FA and MD values in FD patients and controls. The level of statistical significance was set at two tailed $\alpha=0.05$.

\section{RESULTS}

The age dependent WMLs load in patients clearly exceeded those of controls (see table 2). There were significant correlations between WMLs load and mean global MD $\left(\mathrm{r}_{\mathrm{s}}=0.558, \mathrm{p}=0.004\right)$ and FA $\left(\mathrm{r}_{\mathrm{s}}=-0.493, \mathrm{p}=0.012\right)$ of the white matter and between age and mean global MD of the white matter $\left(r_{s}=0.431, p=0.031\right)$ in FD patients. However, these correlations did not remain significant when age and WMLs load were alternatively excluded using partial correlation. Only age itself correlated significantly with WMLs load $\left(\mathrm{r}_{\mathrm{s}}=0.661, \mathrm{p}<0.001\right)$ of FD patients. Furthermore, the association of clinical symptoms (angiokeratoma, neuropathic pain, cerebrovascular events, cardiovascular and renal dysfunction) and the imaging parameters of the patient group was analysed using univariate analysis with age as the covariate (ANOVA). None of the analysed parameters (see table 1) had a significant effect on the WMLs load or on DTI parameters in FD patients. Moreover, using a binary logistic regression, neither WMLs load nor global MD or FA values were found to be predictive for cerebrovascular events in our study group.

Using voxel based DTI analysis, MD differed significantly $(\mathrm{p}<0.001$, uncorrected) between FD and controls (table 3, fig $1 \mathrm{~A})$. MD values were significantly increased, mainly in regions of the frontal, temporal, central and parietal white matter (table 3, fig $1 \mathrm{~A}$ ).

In gray matter regions, significant $\mathrm{MD}$ increases were detected only in the posterior thalamus bilaterally $(p<0.001$, uncorrected; table 3, fig 1A). Thalamic diffusivity elevation remained significant after exclusion of two male patients who showed pulvinar hyperintensities on Tl weighted images and three patients with visible thalamic lesions on $\mathrm{T} 1$ or $\mathrm{T} 2$ weighted images.

Even FD patients without significant WMLs $(n=18)$ showed a significant $\mathrm{MD}(\mathrm{p}<0.001$, uncorrected; table 3, fig $1 \mathrm{~B})$

Table 3 Talairach space derived distribution of white matter regions with increased mean diffusivity $(p<0.001$ uncorrected) in patients with Fabry disease with and without significant white matter lesions compared with age matched controls

\begin{tabular}{|c|c|c|c|c|c|}
\hline \multicolumn{3}{|c|}{ Centroid voxels } & \multirow[b]{2}{*}{$\mathrm{T} \dagger$} & \multirow{2}{*}{$\begin{array}{l}\text { Cluster } \\
\text { size }\end{array}$} & \multirow{2}{*}{$\begin{array}{l}\text { Location: adjacent gray matter region } \\
\text { (Brodmann area } \neq \text { ) }\end{array}$} \\
\hline$x$ & $\mathbf{Y}$ & $\mathbf{Z}^{*}$ & & & \\
\hline \multicolumn{6}{|c|}{ Patients with WMLs } \\
\hline-25 & -14 & 28 & 4.76 & 13453 & Left sub-lobar sub-gyral (13) \\
\hline 51 & -28 & -14 & 4.64 & 1941 & Right temporal lobe sub-gyral (21) \\
\hline 24 & -51 & 34 & 4.44 & 1203 & Right parietal lobe sub-gyral (31) \\
\hline-43 & -58 & -3 & 4.43 & 1198 & Left temporal lobe sub-gyral (37) \\
\hline 12 & -43 & 25 & 4.38 & 432 & Right limbic lobe cingulate gyrus (31) \\
\hline-13 & -23 & -1 & 4.28 & 266 & Left thalamus ventral posterior medial nucleus \\
\hline-46 & -17 & -22 & 4.13 & 1548 & Left temporal lobe sub-gyral (20) \\
\hline 30 & -31 & 23 & 4.08 & 1265 & Right sub-lobar sub-gyral (13) \\
\hline-18 & 38 & 20 & 4.01 & 2336 & Left frontal lobe medial frontal gyrus (32) \\
\hline 14 & 46 & 26 & 3.99 & 269 & Right frontal lobe superior frontal gyrus (9) \\
\hline 15 & -22 & -2 & 3.93 & 106 & Right thalamus ventral posterior medial nucleus \\
\hline \multicolumn{6}{|c|}{ Patients without WMLs } \\
\hline-34 & -38 & 27 & 4.35 & 3622 & Left sub-lobar sub-gyral \\
\hline 17 & 55 & 31 & 4.15 & 515 & Right frontal lobe superior frontal gyrus (9) \\
\hline 52 & -27 & -15 & 4.07 & 231 & Right temporal lobe middle temporal gyrus (21) \\
\hline-14 & -87 & -7 & 3.71 & 196 & Left occipital lobe lingual gyrus (18) \\
\hline
\end{tabular}

WML, white matter lesion.

*Talairach coordinates; †only T scores $>3.6$ presented; łnearest identified Brodmann area of significantly increased mean diffusivity. 


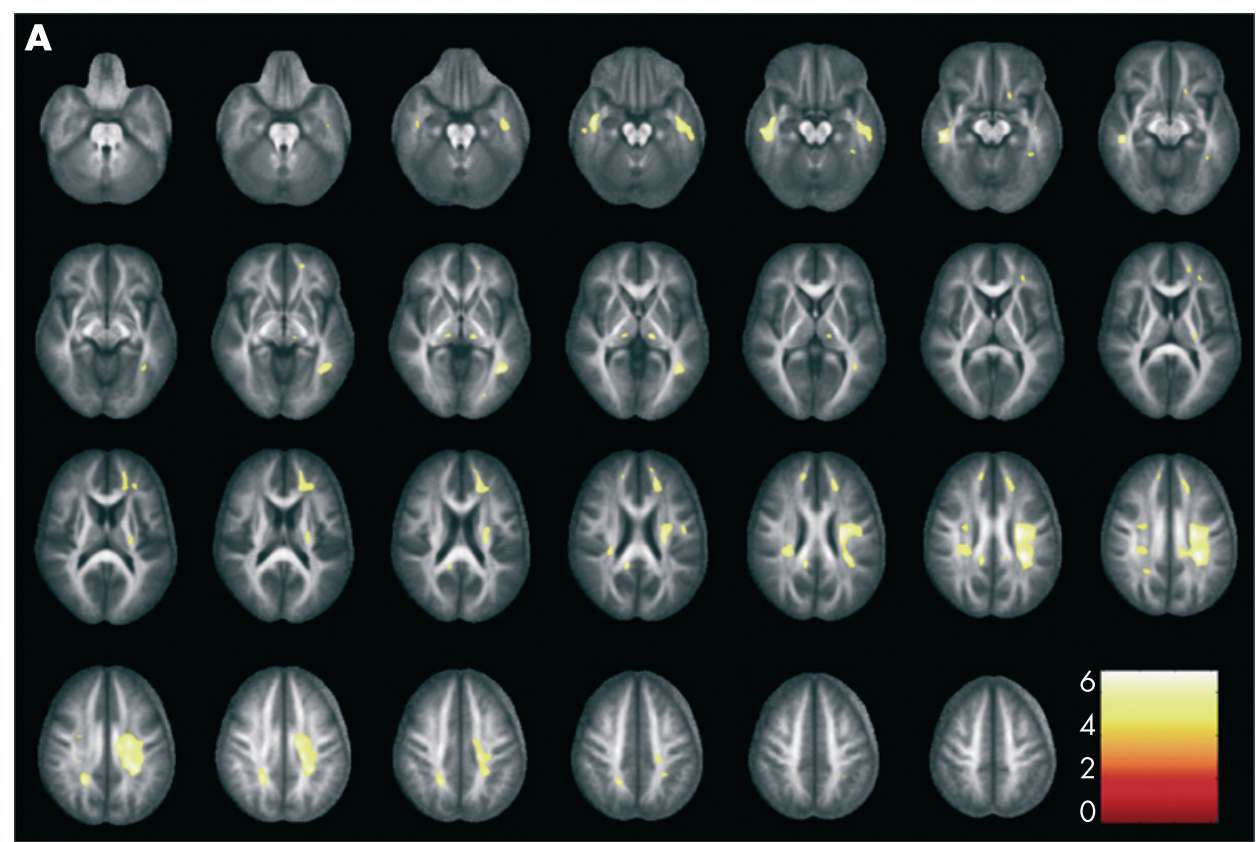

Figure 1 Voxel based comparison of mean diffusivity (MD) between patients with Fabry disease (FD), with (A) and without (B) significant white matter lesions (WMLs), and age matched controls. Significant MD elevations are projected on transverse sections ( $3 \mathrm{~mm}$ distance) of the fractional anisotropy template. (A) MD values were significantly increased mainly in regions of the frontal, temporal, central and parietal white matter ( $p<0.001$, uncorrected). In gray matter regions, significant $M D$ increases were detected in the posterior thalamus bilaterally. (B) FD patients without significant WMLs $(n=18)$ showed significant $M D$ increases in similar locations but with a lower total cluster size.

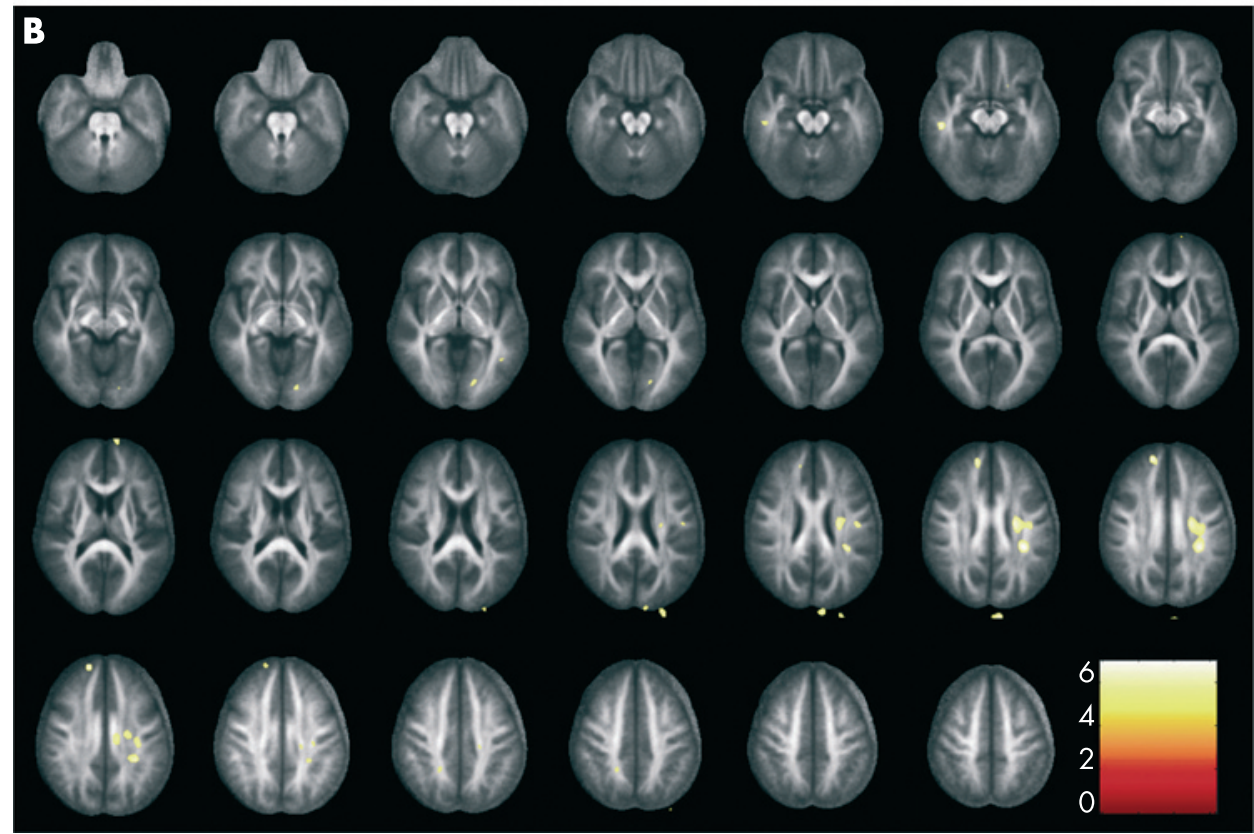

increase in similar locations but with a lower total cluster size of approximately $23 \%$.

In contrast with the significant diffusivity alterations, no anisotropy (FA) differences were detected comparing gray and white matter segmented FA maps of FD patients and controls.

\section{DISCUSSION}

In the present study, we used DTI and voxel based analyses to quantify brain structural changes in FD.

The most prominent finding was a marked disseminated increase in cerebral white matter diffusivity (MD) in patients with FD while FA did not differ significantly between patients and controls.

Comparably with our previously published ROI based DTI findings, increases in diffusivity were pronounced in the periventricular white matter. Crutchfield and colleagues ${ }^{7}$ also found that most cerebral abnormalities in FD were located in the periventricular deep white matter. These areas are supplied by long perforating arteries of small calibre. Thus our results support the assumption of pronounced and relevant dysfunction of these vessels in FD. In contrast, other studies suggested that cerebral abnormalities were pronounced in the posterior artery territory, ${ }^{9}$ mainly because of cerebral macroangiopathy, which is not supported by our data.

In agreement with our previously published ROI based DTI findings and other published brain imaging studies, ${ }^{56832}$ voxel based assessed diffusivity elevations were still significant after exclusion of seven patients with a significant WMLs load. This indicates that measurable elevations in interstitial water content and myelin rarification as degenerative changes of the brain tissue in FD probably emerge before WMLs are detectable on conventional images.

Moreover, we found significant increases in diffusivity in the posterior part of the thalamus, suggesting structural vulnerability, at least in this area of the posterior circulation, in FD. Increased signal intensity on $\mathrm{Tl}$ weighted images has been repeatedly described in the pulvinar regions ("pulvinar sign") ${ }^{33} 34$ However, as the diffusivity differences did not disappear after excluding two patients who showed the "pulvinar sign" on Tl weighted images, MD elevations and pulvinar sign in FD do not seem to refer to the 
same pathophysiological mechanism. One can speculate that increased cerebral blood flow in the posterior artery territory predisposes to thalamic calcification and degenerative tissue rarification or increase in thalamic water content that is eventually represented as a Tl weighted signal increase in the pulvinar. ${ }^{33} 34$ On the other hand, retrograde and anterograde deafferentation due to remote cerebral lesions such as WMLs might be a cause for distinct MD elevations in the thalamus ${ }^{35}$ that need further investigation.

Using voxel based measurements, we were able to analyse the entire gray matter compartment. Interestingly, apart from widespread white matter affection, no diffusivity changes were found in the gray matter. This finding supports the theory that CNS dysfunction in FD is mainly due to insufficiency of the long perforating arteries resulting predominantly in structural changes of the periventricular deep white matter. ${ }^{51336}$ Conversely, it is known from autopsy studies that glycolipid deposition is not only found in blood vessel walls but also in specific small neuronal populations in the CNS in FD. ${ }^{37}{ }^{38}$ These pathological intraneuronal changes that are not known to have a functional correlate may potentially go undetectable with DTI.

In contrast with the marked diffusivity (MD) changes, white matter anisotropy (FA), indicating disturbances of nerve fibre integrity, was not significantly altered in FD.

Quantification of global MD and FA values of the white matter compartment revealed no significant association with age, clinical symptoms or WMLs load. Only age, but not the clinical symptoms, effected WMLs load significantly. Thus our findings support previous suggestions of the close relation between age and the early progression of WMLs load in FD. ${ }^{7}$ Unexpectedly, cerebrovascular events as well as cerebrovascular risk factors, such as renal and cardiac dysfunction, were not significantly related to the brain structural disturbances, which is probably because of the small study group. The previous finding of an increased WMLs load in young stroke patients with FD compared with other young cryptogenic stroke patients indicates an association between early structural alterations and ischaemic events in FD. ${ }^{3}$

Apart from the small study sample size, one limitation of the present study is artefacts due to misregistration, low signal to noise ratio and potential volume contamination of the voxel based DTI approach. These affect FA more than MD values using a template based method. Thus in addition to the assumption that an increase in interstitial water content within the white matter in FD results in an increase in diffusivity (MD) while fibre integrity (FA) is not yet significantly disturbed, the dissociation of MD and FA findings could also be influenced by these technical limitations. For more conservative restriction of partial volume artefacts due to CSF contamination, data processing was additionally refined using a threshold approach in DTI maps according to previous studies. ${ }^{27-29}$

\section{CONCLUSION}

Voxel based diffusion tensor analyses of FD patients revealed significant elevations in brain tissue diffusivity, mainly in the periventricular deep white matter. This pattern of diffusivity alterations that is probably caused by microangiopathy was detected independently of visible WMLs in FLAIR weighted magnetic resonance images. Additionally, MD elevations were detected in the posterior region of the thalamus.

As an investigator independent magnetic resonance technique, voxel based DTI analysis demonstrated its favourable sensitive properties in quantifying brain tissue alterations in FD. In the future, longitudinal assessments using our approach could be used to quantify cerebral involvement and its clinical relevance in the course of FD and related disorders. This could be achieved by providing, for example, further information about the predictive value of early ultrastructural diffusivity changes on cerebrovascular events and by monitoring the cerebral effects of enzyme replacement therapy and other treatment options.

\section{ACKNOWLEDGEMENTS}

We thank the patients and volunteers who took part in our study.

\section{Authors' affiliations}

J Albrecht, P R Dellani, P Stoeter, Institute of Neuroradiology, Children's Hospital of the University of Mainz, Mainz, Germany

M J Müller, I Schermuly, A Gerhard, A Fellgiebel, Department of

Psychiatry, Children's Hospital of the University of Mainz, Mainz, Germany M Beck, Centre for Lysosomal Storage Disorders, Children's Hospital of the University of Mainz, Mainz, Germany

Competing interests: None.

\section{REFERENCES}

1 Meikle PJ, Hopwood JJ, Clague AE, et al. Prevalence of lysosomal storage disorders. JAMA 1999;281:249-54.

2 Desnick RJ IY, Eng CM. Galactosidase A deficiency: Fabry disease, 8th edn. New York: McGraw-Hill, 2001.

3 Rolfs A, Bottcher T, Zschiesche M, et al. Prevalence of Fabry disease in patients with cryptogenic stroke: a prospective study. Lancet 2005;366:1794-6.

4 Mehta A, Ricci R, Widmer U, et al. Fabry disease defined: baseline clinical manifestations of 366 patients in the Fabry Outcome Survey. Eur J Clin Invest 2004;34:236-42.

5 Moore DF, Altarescu G, Barker WC, et al. White matter lesions in Fabry disease occur in 'prior' selectively hypometabolic and hyperperfused brain regions. Brain Res Bull 2003;62:231-40.

6 Fellgiebel A, Mazanek M, Whybra C, et al. Pattern of microstructural brain tissue alterations in Fabry disease: a diffusion-tensor imaging study. J Neurol 2006;253:780-7.

7 Crutchfield KE, Patronas NJ, Dambrosia JM, et al. Quantitative analysis of cerebral vasculopathy in patients with Fabry disease. Neurology 1998;50:1746-9.

8 Tedeschi G, Bonavita S, Banerjee TK, et al. Diffuse central neuronal involvement in Fabry disease: a proton MRS imaging study. Neurology 1999;52:1663-7

9 Mitsias P, Levine SR. Cerebrovascular complications of Fabry's disease. Ann Neurol 1996;40:8-17

10 Ginsberg L, Manara R, Valentine AR, et al. Magnetic resonance imaging changes in Fabry disease. Acta Paediatr Suppl 2006;95:57-62.

11 Fellgiebel A, Muller MJ, Mazanek M, et al. White matter lesion severity in male and female patients with Fabry disease. Neurology 2005;65:600-2.

12 Fellgiebel A, Muller MJ, Ginsberg L. CNS manifestations of Fabry's disease. Lancet Neurol 2006;5:791-5.

13 Pantoni L, Garcia JH. Pathogenesis of leukoaraiosis: a review. Stroke 1997;28:652-9.

14 Prins ND, van Straaten EC, van Dijk EJ, et al. Measuring progression of cerebral white matter lesions on MRI: visual rating and volumetrics. Neurology 2004;62:1533-9

15 Nakayama N, Okumura A, Shinoda J, et al. Evidence for white matter disruption in traumatic brain injury without macroscopic lesions. J Neurol Neurosurg Psychiatry 2006;77:850-5.

16 Pfefferbaum A, Sullivan EV. Microstructural but not macrostructural disruption of white matter in women with chronic alcoholism. Neuroimage 2002;15:708-18.

17 Fellgiebel A, Wille P, Muller MJ, et al. Ultrastructural hippocampal and white matter alterations in mild cognitive impairment: a diffusion tensor imaging study. Dement Geriatr Cogn Disord 2004;18:101-8.

18 Beaulieu C, Allen PS. Water diffusion in the giant axon of the squid: implications for diffusion-weighted MRI of the nervous system. Magn Reson Med 1994;32:579-83.

19 Le Bihan D, Mangin JF, Poupon C, et al. Diffusion tensor imaging: concepts and applications. J Magn Reson Imaging 2001;13:534-46.

20 Moore DF, Schiffmann R, Ulug AM. Elevated CNS average diffusion constant in Fabry disease. Acta Paediatr Suppl 2002;91:67-8.

21 Desnick RJ, Brady R, Barranger J, et al. Fabry disease, an under-recognized multisystemic disorder: expert recommendations for diagnosis, management, and enzyme replacement therapy. Ann Intern Med 2003;138:338-46.

22 Basser PJ, Mattiello J, LeBihan D. Estimation of the effective self-diffusion tensor from the NMR spin echo. J Magn Reson B 1994; 103:247-54.

23 Ashburner J, Friston KJ. Voxel-based morphometry-the methods. Neuroimage 2000;11(Pt 1):805-21.

24 Talairach J, Tournoux P. Co-planar stereotaxic atlas of the human brain. New York: Thieme Medical Publishers, 1988.

25 Li X, Li L, Lu H, et al. Partial volume segmentation of brain magnetic resonance images based on maximum a posteriori probability. Med Phys 2005;32:2337-45.

26 Medina D, DeToledo-Morrell L, Urresta F, et al. White matter changes in mild cognitive impairment and AD: A diffusion tensor imaging study. Neurobiol Aging 2006;27:663-72. 
27 Virta A, Barnett A, Pierpaoli C. Visualizing and characterizing white matter fiber structure and architecture in the human pyramidal tract using diffusion tensor MRI. Magn Reson Imaging 1999;17:1121-33.

28 Holtmannspotter M, Peters N, Opherk C, et al. Diffusion magnetic resonance histograms as a surrogate marker and predictor of disease progression in CADASIL: a two-year follow-up study. Stroke 2005;36:2559-65.

29 O'Sullivan M, Barrick TR, Morris RG, et al. Damage within a network of white matter regions underlies executive dysfunction in CADASIL. Neurology 2005:65:1584-90

30 Pfefferbaum A, Sullivan EV. Increased brain white matter diffusivity in normal adult aging: relationship to anisotropy and partial voluming. Magn Reson Med 2003;49:953-61

31 Salat DH, Tuch DS, Greve DN, et al. Age-related alterations in white matter microstructure measured by diffusion tensor imaging. Neurobiol Aging 2005;26:1215-27.

32 Politei JM, Capizzano AA. Magnetic resonance image findings in 5 young patients with Fabry disease. Neurologist 2006;12:103-5.
33 Moore DF, Ye F, Schiffmann R, et al. Increased signal intensity in the pulvinar on T1-weighted images: a pathognomonic MR imaging sign of Fabry disease. AJNR Am J Neuroradiol 2003;24:1096-101.

34 Takanashi J, Barkovich AJ, Dillon WP, et al. T1 hyperintensity in the pulvinar: key imaging feature for diagnosis of Fabry disease. AJNR Am J Neuroradiol 2003;24:916-21.

35 Molko N, Pappata S, Mangin JF, et al. Diffusion tensor imaging study of subcortical gray matter in cadasil. Stroke $2001 ; 32: 2049-54$.

36 Shibata $M$, Ohtani $R$, Ihara $M$, et al. White matter lesions and glial activation in a novel mouse model of chronic cerebral hypoperfusion. Stroke 2004;35:2598-603

37 deVeber GA, Schwarting GA, Kolodny EH, et al. Fabry disease: immunocytochemical characterization of neuronal involvement. Ann Neurol 1992;31:409-15

38 Kaye EM, Kolodny EH, Logigian EL, et al. Nervous system involvement in Fabry's disease: clinicopathological and biochemical correlation. Ann Neurol 1988;23:505-9.

\section{BMJ Clinical Evidence-Call for contributors}

BMJ Clinical Evidence is a continuously updated evidence-based journal available worldwide on the internet which publishes commissioned systematic reviews. BMJ Clinical Evidence needs to recruit new contributors. Contributors are healthcare professionals or epidemiologists with experience in evidence-based medicine, with the ability to write in a concise and structured way and relevant clinical expertise.

Areas for which we are currently seeking contributors:

- Secondary prevention of ischaemic cardiac events

- Acute myocardial infarction

- MRSA (treatment)

- Bacterial conjunctivitis

However, we are always looking for contributors, so do not let this list discourage you.

Being a contributor involves:

- Selecting from a validated, screened search (performed by in-house Information Specialists) valid studies for inclusion.

- Documenting your decisions about which studies to include on an inclusion and exclusion form, which we will publish.

- Writing the text to a highly structured template (about 1500-3000 words), using evidence from the final studies chosen, within 8-10 weeks of receiving the literature search.

- Working with BMJ Clinical Evidence editors to ensure that the final text meets quality and style standards.

- Updating the text every 12 months using any new, sound evidence that becomes available. The BMJ Clinical Evidence in-house team will conduct the searches for contributors; your task is to filter out high quality studies and incorporate them into the existing text.

- To expand the review to include a new question about once every 12 months.

In return, contributors will see their work published in a highly-rewarded peer-reviewed international medical journal. They also receive a small honorarium for their efforts.

If you would like to become a contributor for BMJ Clinical Evidence or require more information about what this involves please send your contact details and a copy of your CV, clearly stating the clinical area you are interested in, to CECommissioning@bmigroup.com.

\section{Call for peer reviewers}

BMJ Clinical Evidence also needs to recruit new peer reviewers specifically with an interest in the clinical areas stated above, and also others related to general practice. Peer reviewers are healthcare professionals or epidemiologists with experience in evidence-based medicine. As a peer reviewer you would be asked for your views on the clinical relevance, validity and accessibility of specific reviews within the journal, and their usefulness to the intended audience (international generalists and healthcare professionals, possibly with limited statistical knowledge). Reviews are usually 1500-3000 words in length and we would ask you to review between 2-5 systematic reviews per year. The peer review process takes place throughout the year, and our turnaround time for each review is 10-14 days. In return peer reviewers receive free access to BMJ Clinical Evidence for 3 months for each review.

If you are interested in becoming a peer reviewer for BMJ Clinical Evidence, please complete the peer review questionnaire at www. clinicalevidence.com/ceweb/contribute/peerreviewer.jsp 